\title{
Research on the Construction Process of a Steel Truss Arch Bridge Based on BrIM
}

\author{
Haoze $\mathrm{Li}^{1}$, HuaiRui Hong ${ }^{1}$, Yanjun $\mathrm{Li}^{1}$ and Lei Wang ${ }^{1 *}$ \\ ${ }^{1}$ School of Civil Engineering and Architecture, University of Jinan, Jinan 250022, China.
}

\begin{abstract}
This paper combined BrIM (Bridge Information Modeling) technology with the bridge engineering to parameterize the information of a through type truss arch bridge, and BIM technology is used for information management and construction process management. Firstly, the information such as the size and material of each member of the truss arch bridge was input to the family library to construct the threedimensional model corresponding to the plane and elevation information, which could be used for the drawing review. Then the Navisworks roaming model was used for collision check, the design development phase of the construction drawings were achieved to component geometry and the detailed tables in Revit was used to manage the materials. At last, TimeLiner in Navisworks was utilized to design the construction process so Navisworks could simulate the construction process. It is found that BrIM technology of Revit combined with Navisworks can effectively improve the communication efficiency of all side such as construction, supervision, design and construction, facilitate the industrial prefabrication of components, reduce the component collision before the construction phase, control the schedule of construction and optimize the construction process.
\end{abstract}

\section{Introduction}

The theory of BIM (Building Information Modeling) was firstly proposed by professor Chunk Eastman ${ }^{[1]}$ in the 1970s. Then the world famous software companies Autodesk and Bentley perfected the concept of BIM and developed its theory. Compared with traditional twodimensional graphic design, BIM technology has unique advantages in $3 \mathrm{D}$ architectural design, time and cost, visualization, product delivery, etc ${ }^{[2]}$. Since 2003, Heikkila $\mathrm{R}^{[3]}$ et al of Finland has carried out a research project called "Intelligent Bridge" which applied 3D technology to bridges with conventional sections or common prestressed T-sections. The application of BIM in bridge engineering is called BrIM (Bridge Information Modeling), which can be implemented in the whole life cycle of bridges. Although BrIM developed slowly in foreign countries these years, it has gradually become a powerful tool for bridge construction and industrial production ${ }^{[4]}$.

As the introduction of BIM technology to China was very late, res ns have been mainly focusing on the buildings but few in bridges for the past decade. In 2011, Chiu $\mathrm{C} \mathrm{T}^{[5]}$ et al in Taiwan established a cloud system for a steel bridge based on BIM and performed a 4D simulation of the hoisting construction. In 2014, Zou Yang ${ }^{[6]}$ carried out BIM design on a prestressed rigid continuous bridge and a small box girder bridge, and researched implementary framework of BrIM during the design and construction phases. In 2016, Shen Haihua ${ }^{[7]}$ et al established an integrated information management platform in bridge construction and maintenance for Yushan Bridge. In 2018, Li Zeyu ${ }^{[8]}$ et al established a BIM parametric model for a frame bridge based on Revit and Civil3D, and realized a three-dimensional dynamic roaming display with Lumion. In China, the mainstream of BIM software is based on the buildings and imported from abroad, and the supports for the domestic bridge specification is very limited. While the types of bridges are various, the construction variances are large, and the construction methods are complex, there are only a few applications of BrIM technology in China. Based on Revit and Navisworks, this paper established a threedimensional model and simulated the construction process of a steel truss arch bridge, which provides some references for the application of BrIM in this type of bridge.

\section{Bridge Overview}

\subsection{A steel trussed arch bridge in Luoyang}

The bridge across the Luohe River is a through type steel truss arch bridge, which located in Luoyang East Ring Road. The bridge situates in an important transportation hub and is graceful designed to coordinate with the surrounding landscape. This bridge started construction in December 2016 and opened to traffic in trial in January 2018. With the total length of $1,444 \mathrm{~m}$, the part crossing the Luohe River is $320 \mathrm{~m}$. The main span is a

*Corresponding author's e-mail: WANG Lei, cea_wangl@ujn.edu.cn 
through type steel truss arch with the length of $160 \mathrm{~m}$ and width of $47 \mathrm{~m}$. The span is novel in structure and difficult in construction, which made it the controlling bid section. The main arch consists of 4 trusses and 4 groups of wind braces. Each side has two trusses with spacing of $2.0 \mathrm{~m}$. The joint spacing is $6 \mathrm{~m}$ linked by box beams. The total weight of the steel components of the main span is 6,900 tons, among which the whole hoisting segment reaches 1,660 tons. The highest point of the rib is approximately $40 \mathrm{~m}$ from the deck, and the BrIM technology operates on the main span (as in Figure.1).

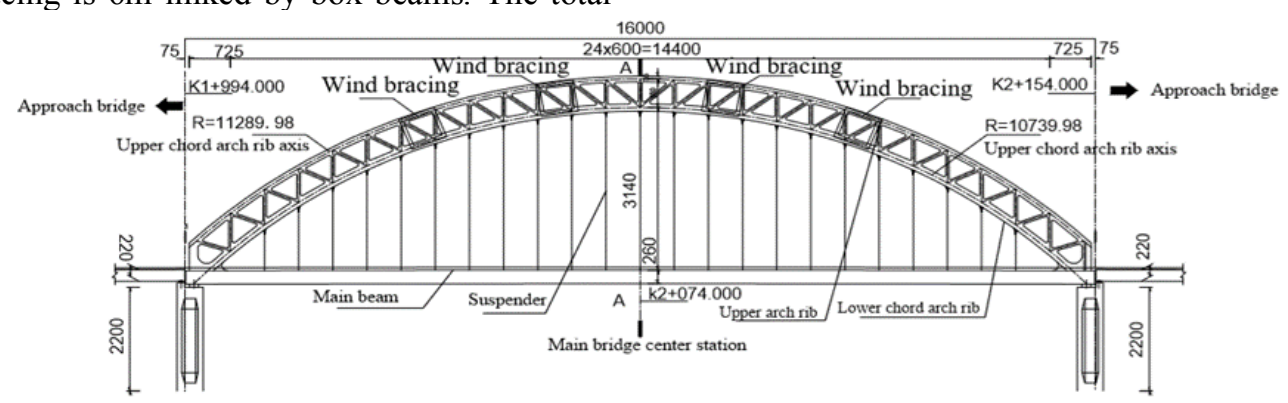

Fig. 1 Main bridge main span layout (unit: $\mathrm{cm}$ )

\subsection{Problems existed in bridge construction}

Just beside this bridge, there is an old bridge called Licheng bridge only 4 meters to it. (as in Figure.2). In order to meet the large demand for traffic, the old bridge could not be demolished or occupied. So the layout of the construction site for the new bridge and the moving and occupation of the large crane were severely restricted. If the gantry crane was used according to the conventional construction method, the cost would be very large because of the complicated trestle of the gantry crane track and the especially solid foundation. The main arch is about 60 meters from the ground and has the width of 47 meters. The gantry crane is larger and has high unsafe factors. The construction process needs to install a part of prefabricated bridge decks in advance, which will increase the work on the key lines of the project and increase the uncertainty factors. This is not conducive to the control of construction progress.

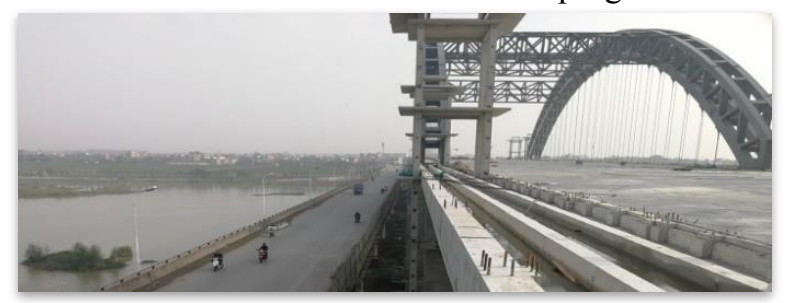

Fig. 2 Location of the truss arch bridge and Bridge of Licheng

The above problems have caused the following difficulties in the construction of the truss arch bridge such as temporary support system layout and calculation, hoisting scheme design comparison on site, steel structure segmentation and segment over-limit transportation, in-plant processing and pre-assembly of arch ribs. BrIM technology can meet the requirements of engineering management, not only improve the installation accuracy of steel arch rib, but also guarantee the safety and reliability of installation and promotion.

\section{Application of BrIM in a truss arch bridge}

\subsection{Establishment of the family library}

Using Revit modeling to build component family libraries can achieve design standardization and improve the model efficiency. Firstly, the information model of all bridge members is established including bridge longitudinal beam, crossbeam, secondary crossbeam, secondary longitudinal beam, arch rib upper chord and lower chord etc. The detailed data information such as size, material and component code is input, and the truss arch bridge family library is established (as in Figure.3). Then the elevation grid of the bridge is established, and all the component families such as piers, caps, longitudinal beams, crossbeams, deck plates and steel truss arch ribs, etc. are loaded into the Revit structure template. The corresponding model family is linked in the plane and steel truss arch bridge model is assembled (as in Figure.4). Finally, after all the families are loaded and assembled, the 3D model is used to observe whether the height of the assembly of the bridge is the corresponding elevation. If there is an error, it must be moved to the corresponding elevation.

Standardized model data is used in the whole process and the accuracy of data such as material, height, length and width is ensured through information parameterization. For these standardized model components, when a component parameter information (as in Figure.4) needs to be changed, the component information is modified in the edit type dialog box and the model information will change accordingly, so that the component information works together and the modeling efficiency is improved. 

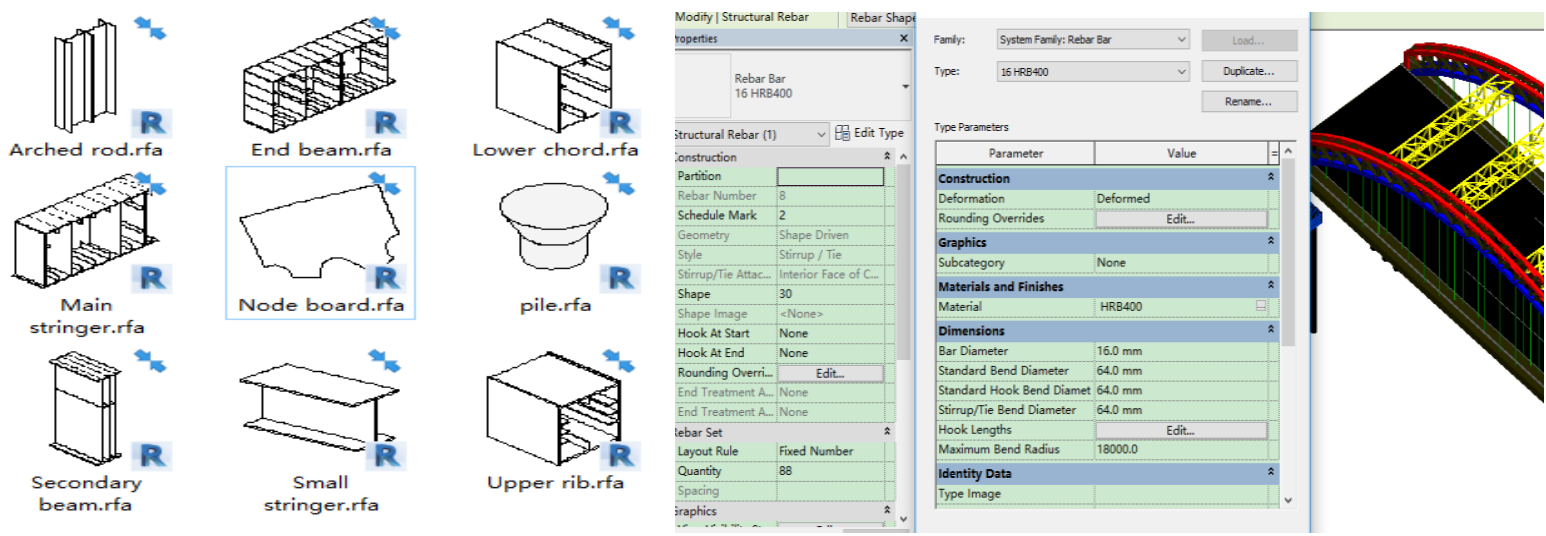

Figure.3 Family library

Figure.4 Parameterization information of the arch bridge

\subsection{Drawing review}

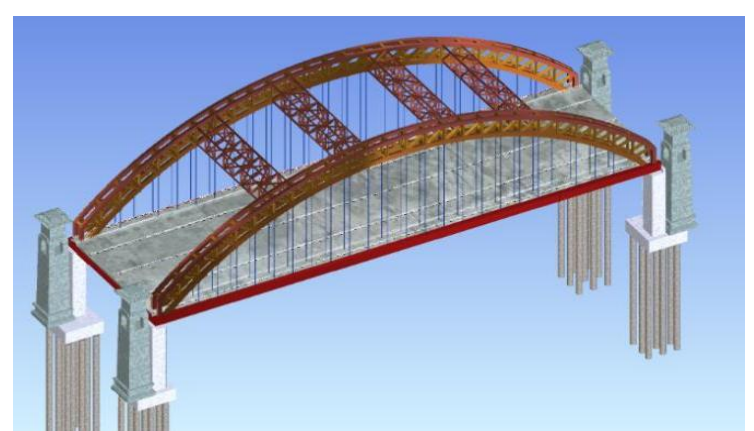

Figure. 5 The three-dimensional model and the design drawing of the truss arch bridge

At the present stage, most of the drawing review in China is examined and inspected on the plane drawings. It is a complicated and heavy work that is needed many units such as construction units, supervision units, design units, construction units, etc., to participate together at the same time and the same place. The three-dimensional lofting in BrIM technology is used to find the design problem in the modeling stage, making the reviewing process more intuitive, and the model can be jointly reviewed by multiple units in different locations. In the process of drawing review, the three-dimensional model of the truss arch bridge (as in Figure.5) is greatly improved, and an ideal state is achieved in deepening construction and coordination.

\subsection{Collision check}

3D visualization technology of BrIM can dynamically check the model in real time and discover problems in the design through Navisworks 3D roaming. In the bridge modeling process, the corresponding NWC format is exported after the map is designed by Revit. Then the model information is imported into Navisworks and the three-dimensional model is subjected to roaming observation and collision check (as in Figure.6), so that

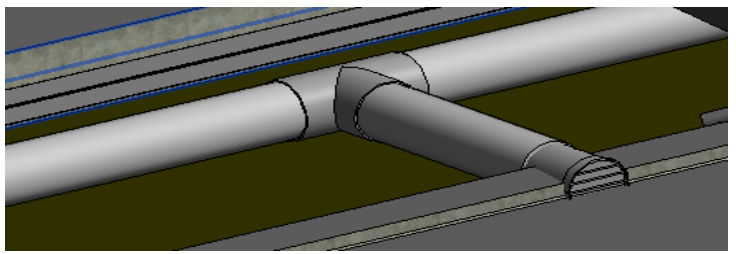

Figure. 6 Collision check for a node

the problems of mistakes, leaks, bumps, collisions, etc. that cannot be avoided under the traditional twodimensional design can be solved well. The conflicting part of the structural inspection is submitted to the design unit to avoid the "fighting" state of the bridge structure and the pipe-series line, and the relevant node large sample drawing can continue to be deepened in detail.

\subsection{Geometry of construction drawings}

Through the BrIM technology, the geometry of the component is achieved (as in Figure.7), so that the worker can process the steel structural member with reference to the three-dimensional model in the production process, which is intuitive and simple. The component error can be reduced at the production source, and the production efficiency and accuracy of the components will be improved in the engineering. Sizeaccurate components make installation more precise during construction and achieve higher quality requirements. BrlM decomposes and digitizes each component to make the number of components accurate which can be achieve industrial prefabrication (Figure.8) and avoid waste during construction. 


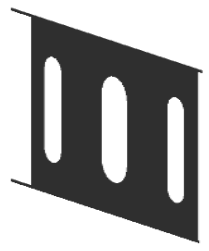

(a) Component of Revit

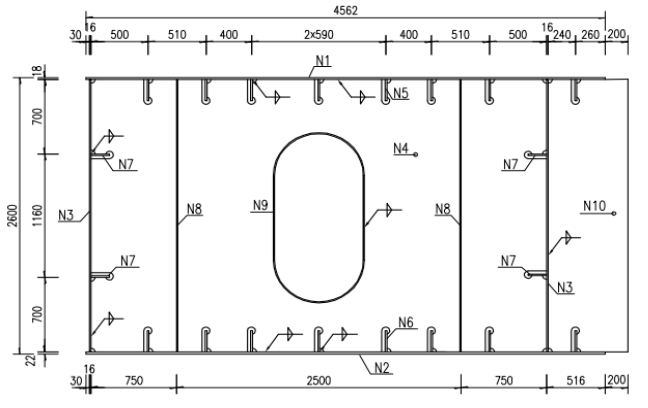

(b) Component drawings

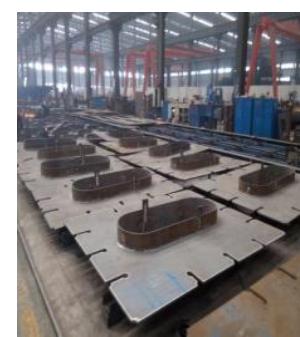

(a) Factory processed components

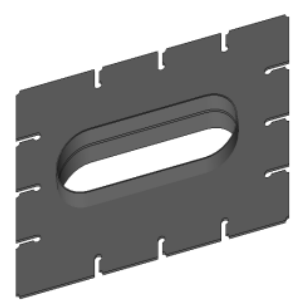

(b) Component model

Fig. 7 Geometry of component

\subsection{Management of materials}

In order to ensure the rational use of materials, management of materials has always been the focus of project management. BrIM technology can combine the construction process with project schedule to carry out detailed procurement plans for related materials, and it can reduce the occurrence of various problems caused by poor management of traditional materials. The detailed tables in Revit can export information such as the name, quantity, and material of the component, etc. This model divided the constructed lever into parts, sub-areas, and classified output material lists (as in Figure.9), including cross-section, length, width, weight, material, model and other data to provide accurate data basis for material procurement. For the cast-in-place reinforced concrete part, the calculation of concrete, steel, etc., were extracted according to the Revit model, which can formulate a reasonable material procurement plan to avoid material scrapping, deviation and waste caused by incorrect material procurement. When you encounter a material change problem, you can quickly correct to export the changed detailed tables, and integrate the material change list which could improve the efficiency of the change program.

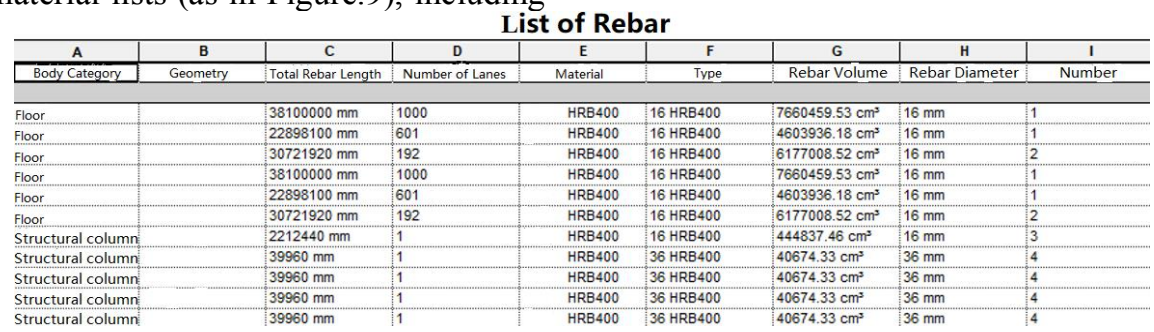

Fig. 9 Revit exported material list

\section{Construction process simulation analysis}

\subsection{Construction schedule control}

The TimeLiner function module in Navisworks can set the construction process of each component of the structure, and correlate the construction schedule with the 3D model of the truss arch bridge to simulate the construction process. Navisworks simulates the construction process by grouping the components in turn. First, we selected the set in Navisworks and group the segment components in the model to be combined with the construction process. Since the bridge model segmented stakeout generation during the Revit modeling process, and the natural segment is formed, so the Revit model file could be imported into Navisworks, and the sections were grouped after checking the items in the "Selection Tree" one-by-one correspondence with the model sections. Then the TimeLiner was used to design the construction process and the group was attached to the task with the "Add Task" function. In order to match the task with the segment quickly, an automatic rule can be established to automatically attach the task with the same name and the selection set. After the construction plan was set on the left side, the Gantt chart is formed on the right side. Gantt charts drawn by other project management software such as Microsoft Project also could be imported directly into TimeLiner (as in Figure.10). By predicting the construction schedule and combining the production capacity and site conditions of the component factory, the quantity of components can be controlled in a planned manner. 


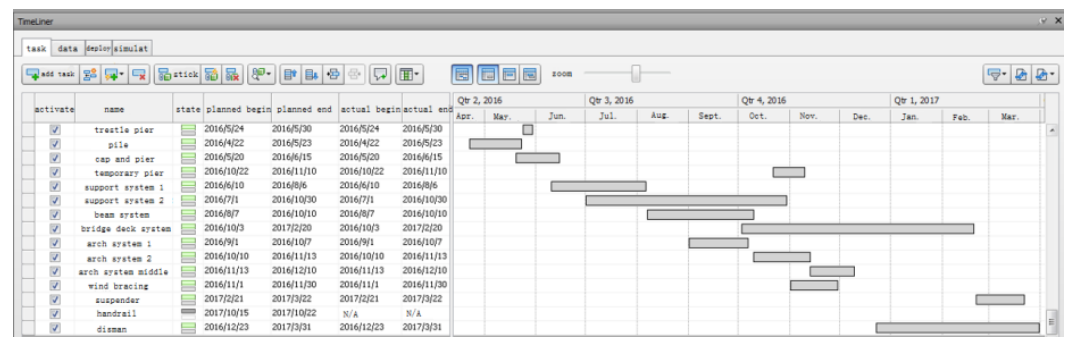

Fig .10 TimeLiner construction process design

\subsection{Simulate of construction process}

Components were managed in the aspects of entry, stacking and handling through the BrIM technology, and each step model is separated and combined. The BIM software was used to formulate the construction plan and simulate the construction process in different stage such as the perfusion of the temporary piles (as in Figure.11), the assembly of steel structural members, the overall lifting of the rib hoisting sections, etc. In the end, the

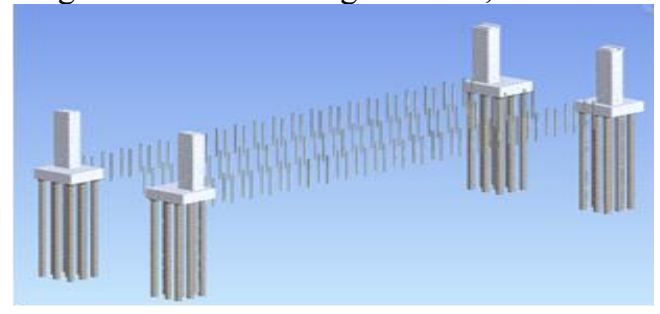

Fig. 11 Tie beam bracket temporary pile

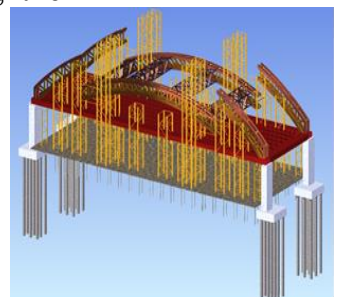

(a) Model diagram

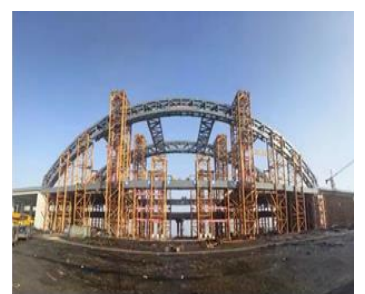

(b) Geometry of site
Figure. 12 Hydraulic jack lifting arch rib jacks (as in Figure.12).

\section{Conclusion}

(1) This paper takes a steel truss arch bridge as an application example, adopts BrIM technology and uses Revit software to create the "family" of each component. The spatial position of each "family" is determined in detail by reference elevation and plane to establish a completed and detailed three-dimensional structural information model for the bridge. It is easy to manage all kinds of material data, guarantee the integrality of component information, and provide the necessary basis for information management.

(2) The Revit model is imported into Navisworks software to generate a three-dimensional roaming model, which can show the overall and detailed structure of the truss arch bridge more vividly and more intuitively than the traditional plane design drawing. The roaming model is used for three-dimensional collision check to find problems before construction, and the scheme is adjusted in time to avoid unnecessary losses.

(3) The construction unit uses BrIM technology to simulate and predict the construction schedule, which can effectively control the schedule of the project. By simulating the construction process, a better construction plan can be chosen to save cost and shorten the construction period. lifting of the arch rib did not use gantry crane, but the combination of high-altitude bulk and assembly: after the steel beam was installed, the bulk rib bulk section was hoisted by a 260 -ton track, the main beam unit at the corresponding position was installed and the intermediate lifting section was assembled at the same time. After the assembly was completed, the lifting section is lifted in place through 8 sets of 350 -ton core-through hydraulic

\section{Acknowledgement}

This study was financially supported by National Natural Science Foundation of China (51408261, 51632003) Shandong Natural Science Foundation Committee (ZR2017JL023). The authors would like to thank reviewers and the Manuscript Editors for their constructive comments on the manuscript.

\section{References}

1. Eastman, C. (1976) General purpose building description systems. Computer-Aided Design , 8(1): 17 26.

2. Hongxue, L., Hongling, G., Yan, G., et al. (2012) Research on Bridge Engineering Design and Construction Optimization Based on BIM, Journal of Engineering Management, (6): 48-52.

3. Heikkila, R., Jaakkola, M., Pulkkinen, P. (2003) Connecting 3-D Concrete Bridge Design to 3-D Site Measurements. The International Symposium on Automation and Robotics in Construction and Mining(ISARC), Eindhoven: ISARC, 259 264.

4. Costin, A., et al. (2018) Building Information Modeling (BIM) for transportation infrastructure Literature review, applications, challenges, and recommendations .Automation in Construction, 94: 257 281. 
5. Chiu, C.T., Hsu, T., Wang, M.T., et a1. (2011) Simulation for steel bridge erection by using BIM tools. Proceedings of the 28th International Symposium on Automation and Robotics in Construction and Mining(ISARC), Seoul: ISARC, 560 563.

6. Yang, Z. (2014) Research on Implementation Framework of Bridge Information Model (BrIM) in Design and Construction Stage. Chongqing :Chongqing Jiaotong University.

7. Haihua, S., Yinhui, W. (2016) Preliminary Study on the Application of Bridge Maintenance Management Based on BIM Highway and motor transport, (04): 280 283.

8. Zeyu, L., Feng, H. (2018) Research on Bridge and Rail Integrated BIM Modeling Method Based on Revit and Civil3D. Railway computer application, 27(5): 43 47. 